\title{
Shortening injection matrix for serial crystallography
}

\author{
Ki Hyun Nam $\mathbb{B D}^{1,2,3}$ \\ Serial crystallography allows crystal structures to be determined at room temperature through the \\ steady delivery of crystals to the X-ray interaction point. Viscous delivery media are advantageous \\ because they afford efficient sample delivery from an injector or syringe at a low flow rate. Hydrophobic \\ delivery media, such as lipidic cubic phase (LCP) or grease, provide a stable injection stream and are \\ widely used. The development of new hydrophobic delivery materials can expand opportunities for \\ future SX studies with various samples. Here, I introduce fat-based shortening as a delivery medium \\ for SX experiments. This material is commercially available at low cost and is straightforward to handle \\ because its phase (i.e., solid or liquid) can be controlled by temperature. Shortening was extruded \\ from a syringe needle in a stable injection stream even below $200 \mathrm{nl} / \mathrm{min}$. X-ray exposed shortening \\ produced several background scattering rings, which have similar or lower intensities than those of LCP \\ and contribute negligibly to data processing. Serial millisecond crystallography was performed using \\ two shortening delivery media, and the room temperature crystal structures of lysozyme and glucose \\ isomerase were successfully determined at resolutions of 1.5-2.0. . Therefore, shortening can be used \\ as a sample delivery medium in SX experiments.
}

Serial crystallography (SX) using an X-ray free electron laser (XFEL) or synchrotron X-ray sources allows crystal structure to be determined without radiation damage or low dose data collection, respectively ${ }^{1-8}$. This technique not only determines the room or cryogenic temperature structure but also enables the time-resolved molecular dynamics to be visualized through pump-probe experiments ${ }^{1-4,9}$. Therefore, SX experiments allow for in-depth analyses of the more biologically reliable crystal structure of macromolecules beyond what is possible with conventional X-ray crystallography. In SX experiments, it is important to deliver the crystal sample serially and stably to the $\mathrm{X}$-ray interaction point ${ }^{10}$. Delivering the sample reliably not only reduces sample consumption but also enables data collection for accurate responses in time-resolved studies ${ }^{11}$. Among the various sample delivery methods, including liquid jet injector ${ }^{12}$, high viscosity injector ${ }^{13,14}$, fixed-target scanning ${ }^{15-17}$, and microfluidics ${ }^{18}$, sample delivery using a viscous medium with an injector or syring $\mathrm{e}^{19-27}$ is widely applied to serial femtosecond crystallography (SFX) or serial millisecond crystallography (SMX), which can successfully determine structure using an XFEL or synchrotron, respectively. The advantage of crystal delivery with viscous extrusion is its feasibility for time-resolved experiments with irreversible systems and the possibility to perform serial crystallography easily at the synchrotron or XFEL facility with a low repetition rate re,25-27. $^{10}$

Based on their physical and chemical properties, sample delivery media can be categorized as either lipidic cubic phase (LCP) $)^{13}$, oil-based ${ }^{19-21}$, or hydrogel-based ${ }^{20-24}$. Among them, LCP and grease, which have hydrophobic characteristics, are widely used as sample delivery media in SX experiments ${ }^{13,19-21}$. LCP (e.g., monoolein-based) is widely applied in the process of membrane protein crystallization and can be used as a sample delivery medium ${ }^{13,28}$. The LCP delivery medium enables the sample to be delivered, even at flow rates of $0.001-0.3 \mu \mathrm{l} / \mathrm{min}^{13,14}$. Moreover, LCP can be used as a sample delivery medium for soluble protein crystal samples and can also be used at atmospheric pressure ${ }^{29}$. The phase of LCP can be changed to the lamellar crystalline phase in a vacuum environment, but the addition of shorter chain lipids can prevent this phase transition ${ }^{13,14}$. However, the phase of LCP can be transformed into lamellar, hexagonal, or sponge, depending on the crystallization or experimental environment, and it is not suitable for use with high concentration of ammonium sulfate ${ }^{14,30}$. Oil-based grease matrices, such as mineral oil grease ${ }^{19}$, synthetic grease Super Lube ${ }^{20}$, and nuclear grade grease $e^{21}$, also provide injection streams and have been applied to various SFX experiments. However, because this material is usually in a semisolid state at room temperature, it is difficult to transfer it to a syringe or other devices, which can cause sample loss during sample preparation ${ }^{19}$. On the other hand, the stability of the injection stream for

${ }^{1}$ Division of Biotechnology, Korea University, Seoul, Republic of Korea. ${ }^{2}$ Institute of Life Science and Natural Resources, Korea University, Seoul, Republic of Korea. ${ }^{3}$ Present address: Department of Life Science, Pohang University of Science and Technology, Pohang, Republic of Korea. email: structures@korea.ac.kr 
typical sample delivery media deteriorates depending on the physical or chemical reactions with the specific crystal sample or crystallization solution. For example, in the case of proteins (e.g., cellulase) that bind or interact with sugar, the crystalline sample may be unstable or dissolved in sugar-based delivery media (e.g., hydroxyethyl cellulose or carboxymethyl cellulose). Thus, it is very important to continuously develop various potential alternative delivery materials ${ }^{27}$.

Shortening consists of fats formulated from oils, and it is a very safe substance used primarily in food production $^{31}$. This material is readily available and has the advantage of being much less expensive than previously reported sample delivery media. In general, shortening has a solid state at room temperature and can be changed to a liquid state by raising its temperature, which is considered useful for sample preparation.

Here, I introduce the characterization and preparation of a shortening injection matrix for serial crystallography. The background scattering in shortening was negligible in data processing and showed similar or low scattering intensity compared to that of LCP. SMX experiments were performed using commercially available shortening, and the room temperature crystal structures of lysozyme and glucose isomerase were determined at resolutions of 1.5-2.0 Å. Shortening can be used for crystal delivery in SFX or SMX experiments.

\section{Results and Discussion}

Characterization and preparation of shortening injection matrix. In this experiment, two types of commercially available oil-based shortening were used, which are denoted as shortening A (composed of palm oil and tallow) and shortening B (composed of palm oils, tallow, and tocopherol). Commercially available shortening can exhibit physically varying melting points $\left(10-52^{\circ} \mathrm{C}\right)^{31-33}$, which is due to the composition of the shortening, the proportions of oils, and the manufacturing process ${ }^{31,32}$. To apply shortening to an injection matrix for an SX experiment, the melting temperatures of shortenings $\mathrm{A}$ and $\mathrm{B}$ were screened in the range of 20 to $40^{\circ} \mathrm{C}$. The results showed that the melting temperatures of shortenings $\mathrm{A}$ and $\mathrm{B}$ were approximately 28.5 and $26.5^{\circ} \mathrm{C}$, respectively.

Next, the sample preparation method using shortening was established for the SX experiment. The crystals embedded in shortenings A and B were prepared by mechanical mixing using a dual syringe setup (Fig. 1). Because the melting temperature of the shortening is below typical body temperature, the liquid phase of the shortening can be achieved by holding the glass vial containing the shortening by hand, but this takes several minutes. To reduce sample preparation time, in this experiment, the glass vial containing the shortening was immersed in hot water $\left(\sim 100^{\circ} \mathrm{C}\right)$ in a beaker for approximately $10-20$ s to obtain its liquid phase (Fig. 1a). The liquid phase shortening was transferred to a $100 \mu \mathrm{l}$ syringe using a pipette and kept at room temperature $\left(23-25^{\circ} \mathrm{C}\right)$ or low temperature in an incubator until solidified (Fig. 1a). The solid state of shortening at room temperature is not hard and does not physically affect the crystalline sample during mixing because of its easily crushed nature. Moreover, the crushed shortenings were not broken due to the viscosity, and the overall mixing process behavior was similar to that of LCP. The crystal suspension was transferred to another syringe using a pipette (Fig. 1b). This syringe was then placed vertically and left for at least 10 minutes (see below). In this case, even if the crystal size is smaller than $20 \mu \mathrm{m}$, it will sink well in the syringe. When the crystals sank to the bottom, the plunger was pushed upwards and the supernatant of the crystal suspension was removed, leaving the crystals (Fig. 1b). Next, two syringes containing shortening and crystals were connected using a coupler and the sample was gently mixed by moving the plunger back and forth until the crystal sample was evenly distributed (Fig. 1c). The mixture sample of crystals and shortening was transferred to a syringe, and the partner syringe with the coupler was removed. After connecting the syringe needle to syringe containing the mixture, the crystals embedded in the shortening were extruded from the syringe needle using a syringe pump (Fig. 1d).

Screening the injection stream of shortening. To determine whether shortening can deliver the crystal sample for SX, stable injection streams for shortenings A and B were investigated using a commercially available syringe and syringe pump. The syringe containing the shortening was connected to the syringe needle with a $168 \mu \mathrm{m}$ inner diameter (ID), and it was vertically installed into the syringe pump. The shortening was extruded from the syringe needle by pushing the syringe plunger using a syringe pump. At low flow rates of less than $200 \mathrm{nl} / \mathrm{min}$, shortening provided a curled injection stream irregularly at the tip of the needle from which the sample came out. To solve this problem, the initial flow rate of the shortening was increased to $3 \mu \mathrm{l} / \mathrm{min}$ and the shortening was extruded for 3-5 s before stopping the syringe pump. The extruded shortening formed a stream of approximately $10 \mathrm{~mm}$ in length, which remained one stream due to its viscosity. When the shortening stream was directed downward in line with gravity, even when shortenings A and B were ejected at a flow rate lower than $200 \mathrm{nl} / \mathrm{min}$, they flowed very steadily in the downward direction (Fig. 2). The main component of shortening is the hydrophobic lipid triglyceride, whereas the crystal mother liquor is hydrophilic. Therefore, the shortening and crystal suspension have different polarities. To achieve a stable shortening injection stream, a suitable ratio of shortening to crystal suspension was investigated. In the mechanical mixing method using a dual syringe setup, the shortening was mixed with lysozyme crystal suspension at ratios of 5:5, 6:4, 7:3, 8:2, and 9:1. When the crystal solution was more than $30 \%$, an unmixed crystal solution was ejected in the middle of the shortening injection stream and disrupted the continuous injection stream. By contrast, less than $20 \%$ of the crystal solution provided a stable shortening injection stream. In order to decrease the ratio of crystal suspension to less than $20 \%$ in the total sample including the shortening, a syringe containing a crystal sample was set up vertically, and when the crystals had settled, the supernatant was removed before mixing with the shortening (Fig. 1c). The percentage of settled crystal suspension was approximately $15-20 \%$ of the total volume, and the shortening containing crystals provided a steady and stable injection stream like LCP injection.

Measurement of background scattering. The X-ray exposed sample delivery media generate background scattering, which can affect the signal-to-noise (SNR) ratio during data processing ${ }^{22,24}$. The background scattering from the delivery medium is one of the most important criteria for delivery medium selection for SX 
a

Glass vial

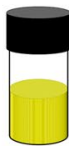

Solid shortening

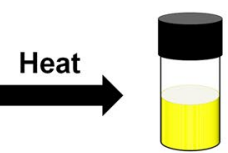

Liquid shortening
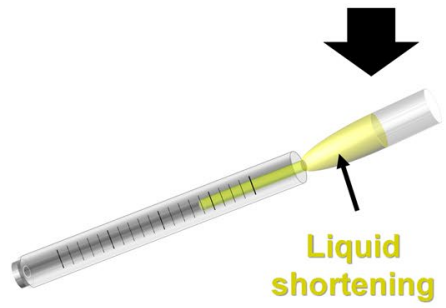

b

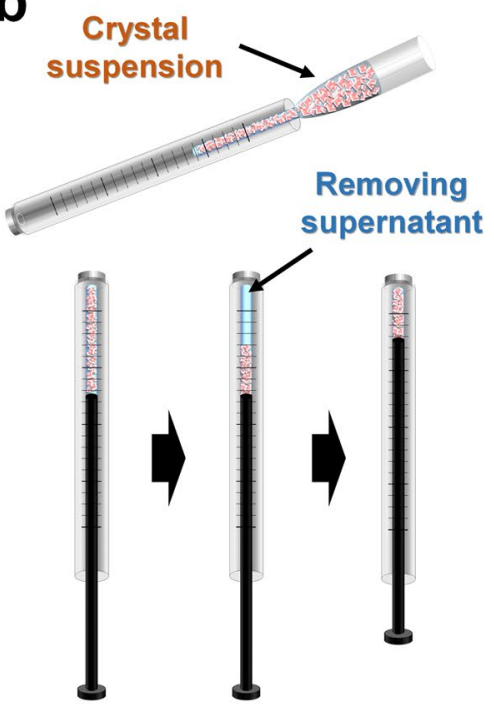

C
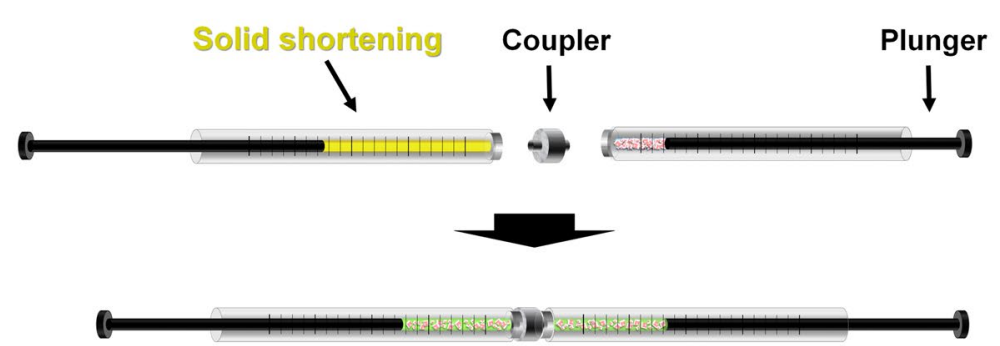

Mixing

d

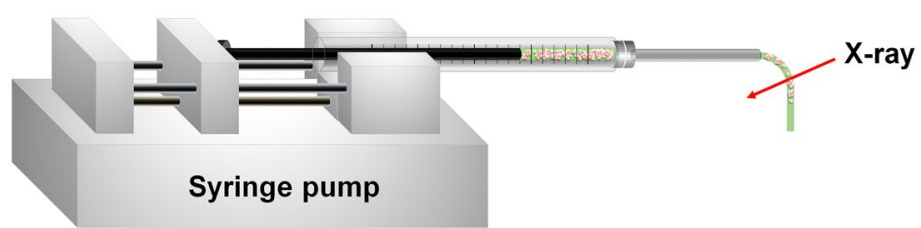

Figure 1. Preparation of shortening injection matrix. (a) Solid shortening in glass vial was immersed in hot water, and the shortening liquid was transferred to a syringe using a pipette. (b) Crystal suspension in syringe was stood vertically. When crystals settled, then supernatant was removed. (c) The syringes containing the shortening and crystals were connected with a coupler and gently mixed. (d) The crystals embedded in the shortening were passed through the syringe needle using a syringe pump and delivered to the X-ray position.
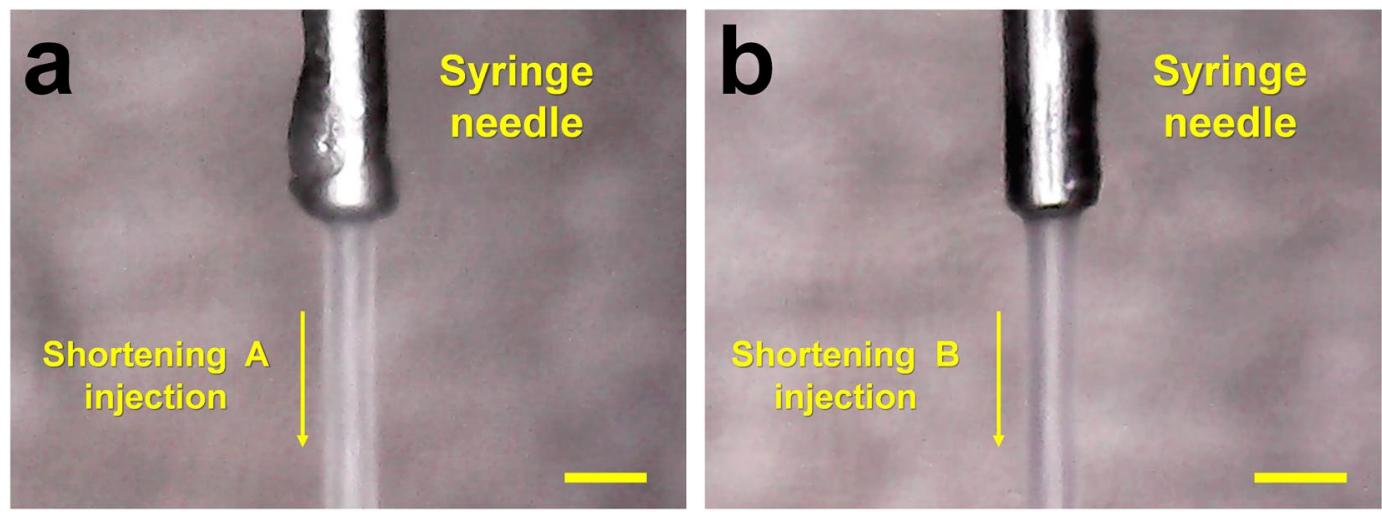

Figure 2. Snapshots of the injection streams of (a) shortenings A and (b) shortening B at a flow rate of $200 \mathrm{nl} /$ min. Scale bar indicates $718 \mu \mathrm{m}$. 


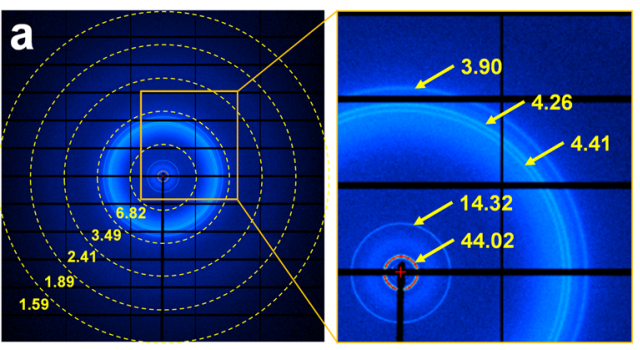

Shortening A
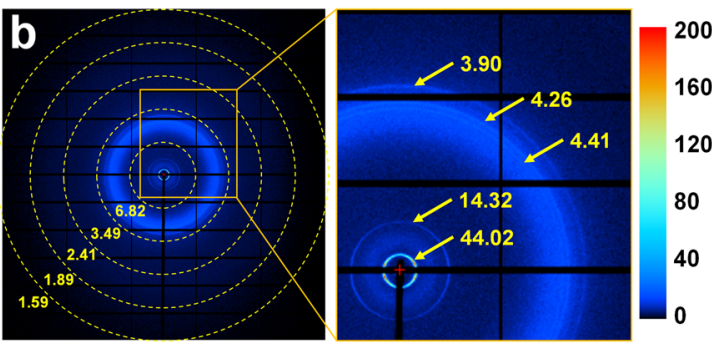

Shortening B

C
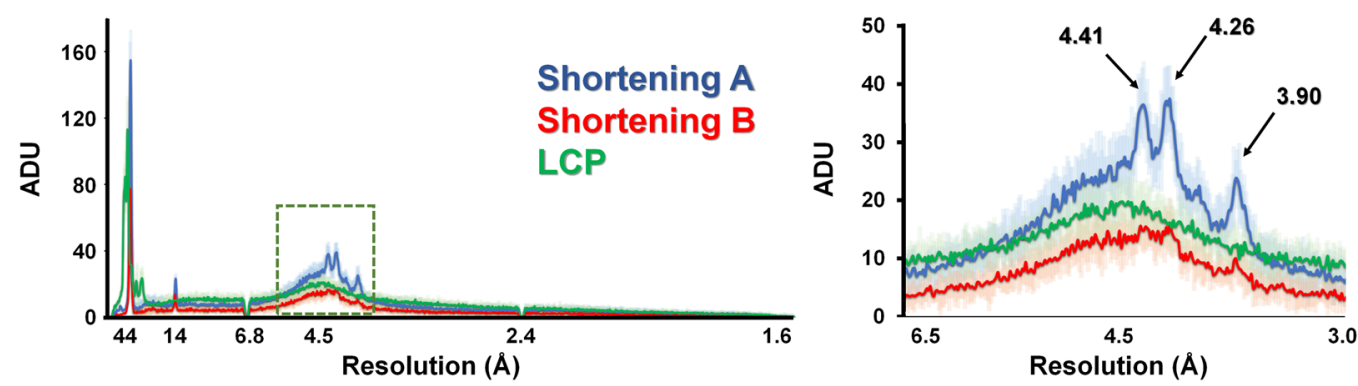

Figure 3. Background X-ray scattering of shortening. Representative detector frames of (a) shortening A and (b) shortening B. (c) 2D profile of the scattering intensities of shortening A (blue), shortening B (red), and LCP (yellow). (right) close-up view of green dotted box. The standard deviations of background intensities of shortening A, shortening B, and LCP are indicated by transparent blue, red, and green region, respectively.

experiments ${ }^{27}$. The analyses of the background scattering of shortenings A and B were compared to the LCP delivery medium consisting of $60 \%(\mathrm{v} / \mathrm{v})$ monoolein. The delivery media were extruded from the syringe needle with a $168 \mu \mathrm{m} \mathrm{ID}$, and they were exposed to X-ray radiation with a photon flux of $1.3 \times 10^{12}$ for $100 \mathrm{~ms}$. For shortening A, five background scattering rings were observed at 3.90, 4.26, 4.41,14.32, and $44.02 \AA$ (Fig. 3a), giving average analog-digital units (ADU) values of $25,37,38,21$, and 155, respectively (Fig. 3c). For shortening B, five background scattering rings were observed at 3.90, 4.26, 4.41,14.32, and $44.02 \AA$ (Fig. 3a), giving average ADU values of $11,16,15,14$, and 59, respectively (Fig. 3c). Therefore, shortening A and shortening B show almost identical background scattering ring patterns in a similar resolution area, whereas the background intensity of shortening $\mathrm{B}$ was 2-3 times lower than that of shortening A (Fig. 3c). The scattering ring patterns from shortenings A and B are considered to be the scattering from the lipid packing in the solid phase. Since the main ingredients of both shortenings are palm oil and tallow, the scattering rings appear at the same resolution. For LCP, two background scattering rings were observed at $4.5 \AA$ and $25-100 \AA$, and the average ADU values were 20 and 110 , respectively (Fig. $3 \mathrm{c}$ ). Analysis of the $2 \mathrm{D}$ profile of background scattering intensity revealed that shortening A had slightly higher background scattering intensity than LCP at approximately $45 \AA$ and $3.0-5.5 \AA$ (Fig. $3 \mathrm{c}$ ). On the other hand, shortening $B$ showed lower overall intensities of background scattering than LCP, excluding the intensity of the scattering ring at approximately $14 \AA$ (Fig. 3c). Standard deviations of background scattering of shortening A, shortening B, and LCP are shown in Fig. $3 \mathrm{c}$ and Supplementary Fig. S1. Therefore shortening can have different background scattering depending on its production process and composition, and the shortening used in this study showed similar or slightly lower background scattering compared to LCP.

SMX using the shortening injection matrix. To demonstrate the application of a shortening injection matrix, SMX experiments using shortenings A and B were performed with the crystals of lysozyme and glucose isomerase as model samples. During sample preparation, the crystallization solution from crystal suspension was removed as much as possible, and mechanical mixing was performed with shortenings A or B using dual-syringe setups. The proportion occupied by crystals was less than $20 \%$ of the total sample volume. In these experiments, the syringe containing the crystals embedded in shortening was installed on the syringe pump in the horizontal direction to avoid beamline interference. The crystal samples were extruded from the syringe needle with a 168 $\mu \mathrm{m}$ ID at a flow rate of $200-300 \mathrm{nl} / \mathrm{min}$ using the syringe pump. In an offline injection study, the shortening injection stream was stably extruded from the syringe at room temperature, but the extruded injection stream of shortening in the experimental hutch at $25^{\circ} \mathrm{C}$ was less viscous like the unsolidified form, making it the injection stream unstable. It is believed that the shortening was partially melted by heat from the beamline device or the camera light. To solve this problem, the internal temperature of the experimental hutch was kept at $20^{\circ} \mathrm{C}$, which provided a very stable shortening injection stream. For shortening A, totals of 64000 and 48000 images were collected for glucose isomerase and lysozyme, respectively. The number of hit images of GI and lysozyme delivered in shortening A were 25626 (hit rate: 40.04\%) and 18926 (39.42\%), respectively. There were 13651 and 15643 final indexed images of glucose isomerase and lysozyme, respectively (Supplementary Figs. S2 and S3). Glucose isomerase delivered in shortening A was processed up to $1.9 \AA$, and the overall SNR, CC, and $\mathrm{R}_{\text {split }}$ were 4.09 , 

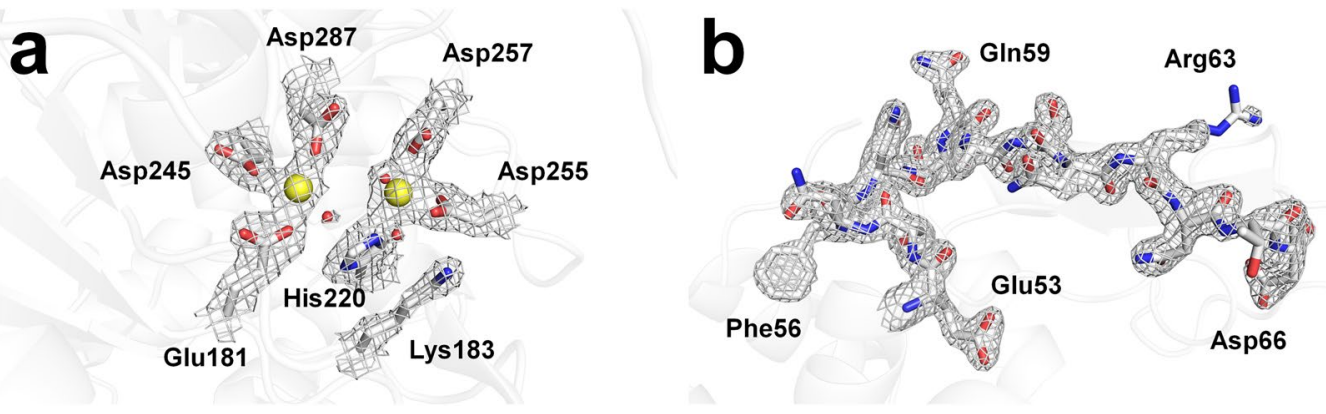

Figure 4. $2 \mathrm{Fo}-\mathrm{Fc}$ electron density maps of (a) catalytic metal binding site in glucose isomerase (light blue mesh, $1.2 \sigma$ ) delivered in shortening A and (b) active site (Glu35 and Asp52) in lysozyme (light blue mesh, $1.5 \sigma$ ) delivered in shortening $\mathrm{B}$.

0.9576, and 19.11, respectively. Lysozyme delivered in shortening A was processed up to $1.8 \AA$, and the overall SNR, CC, and $\mathrm{R}_{\text {split }}$ were 7.87, 0.9937, and 7357, respectively. The final $\mathrm{R}_{\text {work }} / \mathrm{R}_{\text {free }}$ values of glucose isomerase and lysozyme in shortening A were 17.95/21.43 and 17.66/20.53, respectively. For shortening B, totals of 48000 images were collected for glucose isomerase and lysozyme, respectively. The number of hit images of GI and lysozyme delivered in shortening B were 30,418 (hit rate: 63.37\%) and 29,290 (61.02\%), respectively. There were 16522 and 27413 final indexed images of glucose isomerase and lysozyme in shortening B, respectively (Supplementary Figs. S4 and S5). Glucose isomerase delivered in shortening B was processed up to $1.9 \AA$, and the overall SNR, CC, and $\mathrm{R}_{\text {split }}$ were $4.10,0.9669$, and 17.88 , respectively. Lysozyme delivered in shortening $\mathrm{B}$ was processed up to $1.5 \AA$, and the overall SNR, CC, and $\mathrm{R}_{\text {split }}$ were $9.27,0.9936$, and 6.58 , respectively. The $\mathrm{R}_{\text {work }} / \mathrm{R}_{\text {free }}$ values of glucose isomerase and lysozyme derived in shortening B were $17.56 / 20.10$ and $18.49 / 20.69$, respectively.

The glucose isomerase delivered in shortenings A and B showed clear electron density maps for amino acids from Tyr3 to Arg387 (Fig. 4a). The active site of glucose isomerase contains two magnesium binding sites for substrate binding and catalytic function ${ }^{34,35}$. These magnesium binding sites of glucose isomerase are well-defined in the electron density map, and there was no negative fo-fc electron density map counted at $3 \sigma$, indicating there was no significant radiation damage (Supplementary Fig. S6). The glucose isomerase structures delivered in shortenings A and B had high similarity with previously reported glucose isomerase structures delivered using grease (PDB code 4W4Q, Experimental method: SFX) ${ }^{19}$ and nylon mesh-based fixed target scanning (6IRK, SFX $)^{17}$ with a root mean squared deviation of $0.1594-0.2549 \AA$ for C $\alpha$ atoms (Supplementary Fig. S7).

The lysozyme delivered in shortenings A and B showed clear electron density maps for amino acids from Lys19 to Leu147 (Fig. 4b). In lysozyme delivered in shortening A, four disulfide bonds (Cys24-Cys145, Cys48-Cys133, Cys82-Cys98, and Cys94-Cys112) were well-defined, and there was no negative fo-fc electron density map counted at $3 \sigma$, indicating that there was no significant radiation damage (Supplementary Fig. S8a). In contrast, in shortening B, lysozyme delivered three disulfide bonds (Cys24-Cys145, Cys48-Cys133, and Cys82-Cys98) with a partial negative fo-fc electron density map counted at $3 \sigma$ (Supplementary Fig. S8b). Despite the fact that procedure and experimental setup of the SMX experiments using shortening A and B were almost identical, the partial negative fo-fc map of disulfide of lysozyme delivered in shortening B was observed. Further studies will be needed to determine whether negative Fo-Fc is due to radiation damage or its effects on the delivery material. On the other hand, the overall lysozyme structures determined using both shortening A and B are similar to previously reported lysozyme structures as delivered by liquid jet sample injector (PDB code 4ET8, Experimental method: SFX $)^{36}$, droplet injector (5DM9, SFX) ${ }^{37}$, and polyacrylamide $(6 \mathrm{IG} 6, \mathrm{SFX})^{24}$ with a root mean squared deviation of 0.0640-0.1496 ̊ for whole C $\alpha$ atoms (Supplementary Fig. S9). Recently, the crystal structures of lysozyme embedded in LCP and polyacrylamide delivery medium were determined to have resolutions of $1.56 \AA$ and $1.76 \AA$, respectively, using the same crystal sample, sample delivery method, and SMX experiment environment ${ }^{25}$. When comparing lysozyme data using shortening A, shortening B, LCP, and polyacrylamide, it can be seen that there is no significant difference, except for the resolution in terms of data statistics (Supplementary Table 1).

\section{Conclusion}

Here, I reported the characterization and preparation of shortening as a sample delivery medium for SX experiments. SMX was successfully demonstrated using two shortening delivery media. The shortenings used in this experiment have a problem; they are only shipped within South Korea. However, it is expected that similar results will be obtained with an injection matrix using shortenings mainly composed of palm and tallow. In this case, shortening may require a screening procedure before in can be used, as it may exhibit differences in melting temperature, background scattering, and reduction of disulfide bonds depending on its production process and composition. On the other hand, the shortening injection stream may become unstable when the temperature increases; therefore, when conducting SX experiments, the temperature should be kept constant or set below the melting temperature in an experimental hutch or shortening injection environment. In addition, because shortening is mostly fat, lipid-related protein samples may be unstable due to specific or non-specific interactions between crystals and shortening. In this experiment, the shortening delivery media delivered crystals only at atmospheric pressure. However, many SFX experiments are also performed in a vacuum environment; hence, further shortening injection experiments should be performed in vacuum to conduct the SX experiment using shortening. Nevertheless, the advantages of shortening are clear in that it is easy to store and much less expensive 


\begin{tabular}{|c|c|c|c|c|}
\hline \multirow[b]{2}{*}{ Data collection } & \multicolumn{2}{|l|}{ Shortening A } & \multicolumn{2}{|l|}{ Shortening B } \\
\hline & GI & Lysozyme & GI & Lysozyme \\
\hline Wavelength $(\AA)$ & \multicolumn{4}{|l|}{0.9796} \\
\hline Photons/sec ${ }^{\mathrm{a}}$ & \multicolumn{4}{|l|}{$\sim 1.3 \times 10^{12}$} \\
\hline Expose time & \multicolumn{4}{|l|}{$100 \mathrm{~ms}$} \\
\hline Space group & I 222 & $\mathrm{P} 4_{3} 2_{1} 2$ & I222 & $\mathrm{P} 4_{3} 2_{1} 2$ \\
\hline \multicolumn{5}{|l|}{ Cell dimensions $(\AA)$} \\
\hline$a$ & 94.19 & 79.55 & 94.19 & 79.18 \\
\hline$b$ & 99.92 & 79.55 & 99.92 & 79.18 \\
\hline$c$ & 103.25 & 38.51 & 103.25 & 38.34 \\
\hline No. collected images & 64000 & 48000 & 48000 & 48000 \\
\hline No. of hits & 25626 & 18926 & 30418 & 29290 \\
\hline No. of indexed images & 13651 & 15643 & 16522 & 27413 \\
\hline \multirow{2}{*}{ Resolution ( $(\AA)$} & $72.46-1.90$ & $80.00-1.80$ & $72.46-2.00$ & $80.00-1.50$ \\
\hline & $(1.96-1.90)$ & $(1.86-1.80)$ & $(2.07-2.00)$ & $(1.55-1.50)$ \\
\hline Unique reflections & $38725(3832)$ & $11974(1148)$ & $33248(3247)$ & 20406 (1995) \\
\hline Completeness & $100.0(100.0)$ & $100.0(100.0)$ & $100.0(100.0)$ & $100.0(100.0)$ \\
\hline Redundancy & $426.9(292.6)$ & $1451.9(1011.7)$ & $701.6(481.7)$ & $756.6(285.8)$ \\
\hline$I / \sigma(I)$ & $4.09(2.07)$ & $7.87(2.21)$ & $4.10(1.72)$ & $9.27(1.68)$ \\
\hline$R_{\text {split }}{ }^{\mathrm{b}}$ & $19.11(53.97)$ & $7.57(46.32)$ & $17.88(63.03)$ & $6.58(70.95)$ \\
\hline $\mathrm{CC}$ & $0.9576(0.6392)$ & $0.9937(0.8016)$ & $0.9669(0.5778)$ & $0.9936(0.5183)$ \\
\hline $\mathrm{CC}^{*}$ & $0.9891(0.8831)$ & $0.9984(0.9433)$ & $0.9915(0.8558)$ & $0.9984(0.8262)$ \\
\hline Wilson B factor $\left(\AA^{2}\right)$ & 30.85 & 36.24 & 31.57 & 39.72 \\
\hline \multicolumn{5}{|l|}{ Refinement statistics } \\
\hline Resolution $(\AA)$ & $71.80-1.90$ & $56.18-1.80$ & $71.80-2.0$ & $55.98-1.50$ \\
\hline $\mathrm{R}_{\text {factor }} / \mathrm{R}_{\text {free }}(\%)^{c}$ & $17.95 / 21.43$ & $17.66 / 20.53$ & $17.56 / 20.10$ & $18.49 / 20.69$ \\
\hline \multicolumn{5}{|l|}{ B-factor (Averaged) } \\
\hline Protein & 34.02 & 36.88 & 33.63 & 40.33 \\
\hline Ligands & 33.5 & 36.45 & 22.9 & 44.35 \\
\hline \multicolumn{5}{|l|}{ R.m.s. deviations } \\
\hline Bond lengths $(\AA)$ & 0.006 & 0.006 & 0.007 & 0.006 \\
\hline Bond angles $\left(^{\circ}\right)$ & 0.825 & 0.787 & 0.831 & 0.797 \\
\hline \multicolumn{5}{|l|}{ Ramachandran plot (\%) } \\
\hline favored & 96.86 & 98.43 & 97.12 & 99.21 \\
\hline allowed & 2.88 & 1.57 & 2.62 & 0.79 \\
\hline outliers & 0.26 & 0 & 0.26 & 0 \\
\hline
\end{tabular}

Table 1. Data collection and refinement statistics. Highest resolution shell is shown in parentheses. ${ }^{\text {aS }}$. position. ${ }^{\mathrm{b}} R_{\text {split }}=(1 / \sqrt{2})=\frac{\sum_{h k l}\left|I_{h k l}^{\text {even }}-I_{h k l}^{\text {odd }}\right|}{\frac{1}{2}\left|I_{h k l}^{\text {even }}-I_{h k l}^{\text {odd }}\right|}{ }^{\mathrm{c}} R_{\text {work }}=\Sigma|| F_{\text {obs }}|-| F_{\text {calc }}|| \Sigma\left|F_{\text {obs }}\right|$, where $F_{\text {obs }}$ and $F_{\text {calc }}$ are the observed and calculated structure-factor amplitudes respectively. $\mathrm{R}_{\text {free }}$ was calculated as $\mathrm{R}_{\text {work }}$ using a randomly selected subset $(10 \%)$ of unique reflections not used for structure refinement.

than previously reported sample delivery media. The price of shortenings used in this experiment was less than $\$ 10$ for $4.5 \mathrm{~kg}$. (e.g., the price is 45,000,000 times lower than that of LCP). Moreover, the transferal of shortening is straightforward by the temperature control of the solid-liquid phase. In this experiment, large-size lysozyme and glucose isomerase crystals were used for data collection. If small crystals are used, the background scattering of the shortening can potentially affect this, which can be improved by using a narrow injection stream. On the other hand, the shortening should be selected by referring to the comparison of background scattering with LCP, which is widely used in SX experiments using small crystals. Therefore, shortening is a promising delivery material for SX experiments with existing hydrophobic delivery materials.

\section{Materials and Methods}

Crystallization. Glucose isomerase from Streptomyces rubiginosus and lysozyme from chicken egg whites were purchased from Hampton Research (HR7-098) and Sigma-Aldrich (L6876), respectively. Glucose isomerase was supplied as a crystal suspension, and it was used directly for SMX experiments, as previously reported ${ }^{17}$. The lysozyme was crystallized using a previously reported procedure ${ }^{17}$. The crystal sizes of glucose isomerase and lysozyme were less than $60 \times 60 \times 40 \mu \mathrm{m}^{3}$ and $40 \times 40 \times 40 \mu \mathrm{m}^{3}$, respectively. The number density of glucose isomerase and lysozyme were approximately $6.0 \times 10^{5}$ and $5.2 \times 10^{5}$ crystals $/ \mu \mathrm{l}$, respectively.

Characterization of shortening. Shortening A (product name: golden shortening) was purchased from Samyang (Republic of Korea), and shortening B (product name: Combi shooting) was purchased from Ottogi 
(Republic of Korea). Shortening A was composed of palm oil and beef tallow. Shortening B was composed of palm olein oil, palm stearin oil, palm hydrogenated oil, tallow, and d-Tocopherol. The melting temperatures of shortenings A and B were measured on a water bath and judged visually. The solid phase shortening at room temperature was initially transferred to a glass vial using a spatula. During the transfer of the shortening to the syringe, the shortening in the glass vial was immersed in hot water $\left(\sim 100^{\circ} \mathrm{C}\right)$ in a beaker for $10-20 \mathrm{~s}$. Liquid shortening was transferred into a $100 \mu \mathrm{l}$ syringe (Hamilton, $81065-1710 \mathrm{RNR}$ ) using a pipette, and was then left to stand until solid. A syringe needle of 168 ID was connected to the syringe containing the shortening, and the syringe was vertically installed in a Fusion Touch 100 syringe pump (CHEMYX). The syringe plunger was pushed by a mechanical force from the syringe pump ${ }^{25}$ and extruded the sample at a flow rate of $<200 \mathrm{nl} / \mathrm{min}$.

Crystal embedding in shortening. Solid shortening in glass vials was dissolved by soaking in hot water $\left(>100^{\circ} \mathrm{C}\right)$ for 20 seconds. The shortening solution $(50 \mu \mathrm{l})$ was transferred to a $100 \mu \mathrm{l}$ syringe and stored at room temperature until it reached a solid state. The crystal suspension $(20 \mu \mathrm{l})$ was transferred to a $100 \mu \mathrm{l}$ syringe. This syringe was vertically orientated for $10 \mathrm{~min}$. When crystals settled on the bottom, the supernatant was removed using a pipette. The syringes containing the shortening and crystals were connected using a syringe coupler and mixed with the plunger gently moving back and forth more than 30 times. The mixture sample was transferred to a syringe and the emptied partner syringe with the coupler was removed. The syringe containing the crystals embedded shortening was connected with a syringe needle of $168 \mu \mathrm{m}$ ID for SMX experiments.

Data collection. SMX experiments using a shortening injection matrix were performed at the $11 \mathrm{C}$ beamline at Pohang Accelerator Laboratory (Republic of Korea). The temperature and humidity were $20^{\circ} \mathrm{C}$ and $20 \%$, respectively. The X-ray beam size focused by a Kirkpatrick-Baez mirror was approximately 4 (vertical) $\times 8$ (horizontal) $\mu \mathrm{m}^{2}$ (FWHM) at the sample position. The photon flux at the sample position was $1.3 \times 10^{12} \mathrm{photons} / \mathrm{s}$, and the X-ray energy was $12.657 \mathrm{keV}$. The shortening containing crystals was delivered by a syringe pump-based sample delivery system ${ }^{25}$ through a syringe needle of $168 \mu \mathrm{m}$ ID at a flow rate of $200-300 \mathrm{nl} / \mathrm{min}$. Crystals were $\mathrm{X}$-ray exposed for $100 \mathrm{~ms}$. Diffraction images were recorded on a Pilatus $6 \mathrm{M}$ with $10 \mathrm{~Hz}$ readout.

Data processing and structure determination. The hit images with diffraction pattern were filtered using the Cheetah program with the peakfinder8 algorithm ${ }^{38}$. The hit images were indexed and merged using the CrystFEL program ${ }^{39}$. The phase problem of the lysozyme and glucose isomerase was solved by molecule replacement using the Phaser-MR in PHENIX ${ }^{40}$, with the crystal structures of lysozyme (PDB code 6IG6) ${ }^{24}$ and glucose isomerase (PDB code 5ZYD) ${ }^{35}$ used as the search models. The model building and refinement were conducted using Coot $^{41}$ and Phenix.refinement in PHENIX, respectively ${ }^{40}$. The geometry was analysed using MolProbity ${ }^{42}$. Figures were generated using PyMOL (https://pymol.org/). Data-collection and structure refinement statistics are summarized in Table 1

Background scattering analysis. Shortening A, shortening B, and LCP delivery media were passed through a syringe needle with a $168 \mu \mathrm{m}$ ID. Each delivery medium was exposed to X-rays of $1.3 \times 10^{12}$ photons/ flux for $100 \mathrm{~ms}$. The background scattering from $1.6 \AA$ to the beam centre was analysed using the average intensity for 100 images. The background intensities of the delivery media were analysed using ADXV (https://www. scripps.edu/tainer/arvai/adxv.html).

Accession codes. The coordinates and structure factors have been deposited in the Protein Data Bank under the accession code 6KCA (Glucose isomerase-shortening A), 6KCB (Lysozyme-shortening A), 6KCC (Glucose isomerase-shortening B), 6KCD (Lysozyme-shortening B). Diffraction images have been deposited to CXIDB under ID 112 (glucose isomerase-shortening A), 113 (Lysozyme-shortening A), 114 (Glucose isomerase-shortening B) and 115 (Lysozyme-shortening B).

Received: 5 July 2019; Accepted: 6 December 2019;

Published online: 09 January 2020

\section{References}

1. Schlichting, I. Serial femtosecond crystallography: the first five years. IUCrJ 2, 246-255 (2015).

2. Chavas, L. M., Gumprecht, L. \& Chapman, H. N. Possibilities for serial femtosecond crystallography sample delivery at future light sources. Struct Dyn 2, 041709 (2015).

3. Martin-Garcia, J. M., Conrad, C. E., Coe, J., Roy-Chowdhury, S. \& Fromme, P. Serial femtosecond crystallography: A revolution in structural biology. Arch Biochem Biophys 602, 32-47 (2016).

4. Johansson, L. C., Stauch, B., Ishchenko, A. \& Cherezov, V. A Bright Future for Serial Femtosecond Crystallography with XFELs. Trends Biochem Sci 42, 749-762 (2017).

5. Gati, C. et al. Serial crystallography on in vivo grown microcrystals using synchrotron radiation. IUCrJ 1, 87-94 (2014).

6. Nogly, P. et al. Lipidic cubic phase serial millisecond crystallography using synchrotron radiation. IUCrJ 2, 168-176 (2015).

7. Martin-Garcia, J. M. et al. Serial millisecond crystallography of membrane and soluble protein microcrystals using synchrotron radiation. IUCrJ 4, 439-454 (2017).

8. Weinert, T. et al. Serial millisecond crystallography for routine room-temperature structure determination at synchrotrons. Nat Commun 8, 542 (2017).

9. Weinert, T. et al. Proton uptake mechanism in bacteriorhodopsin captured by serial synchrotron crystallography. Science 365, 61-65 (2019).

10. Grunbein, M. L. \& Nass Kovacs, G. Sample delivery for serial crystallography at free-electron lasers and synchrotrons. Acta Crystallogr D Struct Biol 75, 178-191 (2019).

11. Spence, J. C. Approaches to time-resolved diffraction using an XFEL. Faraday Discuss 171, 429-438 (2014).

12. DePonte, D. P. et al. Gas dynamic virtual nozzle for generation of microscopic droplet streams. J Phys D Appl Phys 41, 195505 (2008).

13. Weierstall, U. et al. Lipidic cubic phase injector facilitates membrane protein serial femtosecond crystallography. Nat. Commun. $\mathbf{5}$, 3309 (2014). 
14. Liu, W., Ishchenko, A. \& Cherezov, V. Preparation of microcrystals in lipidic cubic phase for serial femtosecond crystallography. Nat Protoc 9, 2123-2134 (2014).

15. Martiel, I., Muller-Werkmeister, H. M. \& Cohen, A. E. Strategies for sample delivery for femtosecond crystallography. Acta Crystallogr D Struct Biol 75, 160-177 (2019).

16. Hunter, M. S. et al. Fixed-target protein serial microcrystallography with an $\mathrm{x}$-ray free electron laser. Sci Rep 4, 6026 (2014).

17. Lee, D. et al. Nylon mesh-based sample holder for fixed-target serial femtosecond crystallography. Sci Rep 9, 6971 (2019).

18. Calvey, G. D., Katz, A. M. \& Pollack, L. Microfluidic Mixing Injector Holder Enables Routine Structural Enzymology Measurements with Mix-and-Inject Serial Crystallography Using X-ray Free Electron Lasers. Anal Chem 91, 7139-7144 (2019).

19. Sugahara, M. et al. Grease matrix as a versatile carrier of proteins for serial crystallography. Nat Methods 12, 61-63 (2015).

20. Sugahara, M. et al. Oil-free hyaluronic acid matrix for serial femtosecond crystallography. Sci Rep 6, 24484 (2016).

21. Sugahara, M. et al. Hydroxyethyl cellulose matrix applied to serial crystallography. Sci Rep 7, 703 (2017).

22. Conrad, C. E. et al. A novel inert crystal delivery medium for serial femtosecond crystallography. IUCrJ 2, 421-430 (2015).

23. Kovacsova, G. et al. Viscous hydrophilic injection matrices for serial crystallography. IUCrJ 4, 400-410 (2017).

24. Park, J. et al. Polyacrylamide injection matrix for serial femtosecond crystallography. Sci Rep 9, 2525 (2019).

25. Park, S. Y. \& Nam, K. H. Sample delivery using viscous media, a syringe and a syringe pump for serial crystallography. J Synchrotron Radiat 26, 1815-1819 (2019).

26. Berntsen, P. et al. The serial millisecond crystallography instrument at the Australian Synchrotron incorporating the "Lipidico" injector. Rev Sci Instrum 90, 085110 (2019).

27. Nam, K. H. Sample Delivery Media for Serial Crystallography. Int J Mol Sci 20, 1094 (2019).

28. Ishchenko, A. et al. Chemically Stable Lipids for Membrane Protein Crystallization. Cryst Growth Des 17, 3502-3511 (2017).

29. Fromme, R. et al. Serial femtosecond crystallography of soluble proteins in lipidic cubic phase. IUCrJ 2, 545-551 (2015).

30. Botha, S. et al. Room-temperature serial crystallography at synchrotron X-ray sources using slowly flowing free-standing highviscosity microstreams. Acta Crystallographica Section D-Structural Biology 71, 387-397 (2015).

31. Ghotra, B. S., Dyal, S. D. \& Narine, S. S. Lipid shortenings: a review. Food Res Int 35, 1015-1048 (2002)

32. Jennings, B. H. \& Akoh, C. C. Trans-Free Plastic Shortenings Prepared with Palm Stearin and Rice Bran Oil Structured Lipid. Journal of the American Oil Chemists' Society 87, 411-417 (2009).

33. Chin, N. L., Rahman, R. A., Hashim, D. M. \& Kowng, S. Y. Palm Oil Shortening Effects on Baking Performance of White Bread. J Food Process Eng 33, 413-433 (2010).

34. Bae, J. E., Kim, I. J. \& Nam, K. H. Crystal structure of glucose isomerase in complex with xylitol inhibitor in one metal binding mode. Biochem Biophys Res Commun 493, 666-670 (2017).

35. Bae, J. E., Hwang, K. Y. \& Nam, K. H. Structural analysis of substrate recognition by glucose isomerase in $\mathrm{Mn}^{2+}$ binding mode at M2 site in S. rubiginosus. Biochem Biophys Res Commun 503, 770-775 (2018).

36. Boutet, S. et al. High-resolution protein structure determination by serial femtosecond crystallography. Science 337, 362-364 (2012).

37. Mafune, F. et al. Microcrystal delivery by pulsed liquid droplet for serial femtosecond crystallography. Acta Crystallogr D Struct Biol 72, 520-523 (2016).

38. Barty, A. et al. Cheetah: software for high-throughput reduction and analysis of serial femtosecond X-ray diffraction data. J Appl Crystallogr 47, 1118-1131 (2014).

39. White, T. A. et al. CrystFEL: a software suite for snapshot serial crystallography. J Appl Crystallogr 45, 335-341 (2012).

40. Adams, P. D. et al. PHENIX: a comprehensive Python-based system for macromolecular structure solution. Acta Crystallogr D Biol Crystallogr 66, 213-221 (2010).

41. Emsley, P. \& Cowtan, K. Coot: model-building tools for molecular graphics. Acta Crystallogr D Biol Crystallogr 60, 2126-2132 (2004).

42. Chen, V. B. et al. MolProbity: all-atom structure validation for macromolecular crystallography. Acta Crystallogr D 66, 12-21 (2010).

\section{Acknowledgements}

I thank the beamline staff at $11 \mathrm{C}$ beamline at Pohang Accelerator Laboratory for their assistance with data collection. The authors thank Global Science experimental Data hub Center (GSDC) at Korea Institute of Science and Technology Information (KISTI) for computational support. This work supported by a Korea University Grant. This work was funded by the National Research Foundation of Korea (NRF-2017M3A9F6029736).

\section{Author contributions}

K.H.N. performed all experiment and wrote the manuscript.

\section{Competing interests}

The author declares no competing interests.

\section{Additional information \\ Supplementary information is available for this paper at https://doi.org/10.1038/s41598-019-56135-1.}

Correspondence and requests for materials should be addressed to K.H.N.

Reprints and permissions information is available at www.nature.com/reprints.

Publisher's note Springer Nature remains neutral with regard to jurisdictional claims in published maps and institutional affiliations.

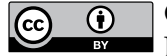

Open Access This article is licensed under a Creative Commons Attribution 4.0 International

License, which permits use, sharing, adaptation, distribution and reproduction in any medium or format, as long as you give appropriate credit to the original author(s) and the source, provide a link to the Creative Commons license, and indicate if changes were made. The images or other third party material in this article are included in the article's Creative Commons license, unless indicated otherwise in a credit line to the material. If material is not included in the article's Creative Commons license and your intended use is not permitted by statutory regulation or exceeds the permitted use, you will need to obtain permission directly from the copyright holder. To view a copy of this license, visit http://creativecommons.org/licenses/by/4.0/.

(c) The Author(s) 2020 